\title{
Estudio de Parámetros de Síntesis de las estructuras zeolíticas Linde Tipo A (LTA) y Faujasita (FAU) X a partir de aluminio post-consumo y diatomita, para la remoción de metales pesados
}

\author{
Jose H. Quintana ${ }^{1}$, Andrea P. Aparicio ${ }^{1}$, Leidy K. Parra² ${ }^{2}$ José A. Henao $^{1, *}$, Carlos A. Ríos ${ }^{3}$ \\ ${ }^{1}$ Grupo de Investigación en Química Estructural (GIQUE), Escuela de Química, Universidad Industrial de Santander, Colombia \\ ${ }^{2}$ Grupo de Investigación en Química Estructural (GIQUE), Escuela Ingeniería Química, Universidad Industrial de Santander, Colombia \\ ${ }^{3}$ Escuela de Geología, Universidad Industrial de Santander, Colombia
}

\begin{abstract}
Resumen
En este estudio, se sintetizaron las zeolitas LTA y FAUJASITA NaX por el método hidrotérmico, usando como material de partida la diatomita, como precursor para la obtención del silicato de sodio y aluminio post-consumo, como precursor de la solución de aluminio. Las zeolitas se caracterizaron por Difracción de Rayos X de Muestras Policristalinas (DRXP), Espectroscopia Infrarroja con atenuación (ATR-IR) y Análisis termogravimétrico (ATG), Calorimetría de Barrido Diferencial (CBD) y Microscopía Electrónica de Barrido (MEB). Las zeolitas sintetizadas presentaron una capacidad de fijación de 4,8 $\mathrm{mmol} / \mathrm{g}$ de $\mathrm{Cu} 2+, 3,4 \mathrm{mmol} / \mathrm{g}$ para el $\mathrm{Cd} 2+$ y 2,3mmol/g de Ni2+, partiendo de los nitratos de estos cationes, lo cual, demuestra el potencial de estos materiales para la remoción de metales pesados.
\end{abstract}

Palabras clave: diatomitas; Aluminio post-consumo; Tratamiento hidrotérmico; Zeolita LTA; Faujasita NaX; Cobre, Níquel y Cadmio.

Study of synthesis parameters of the sodic Linde Tipe A (LTA) and Faujasite (FAU) X zeolite frameworks from post-consumer aluminium and diatomite for removal of heavy metals

\begin{abstract}
In this study, the LTA and faujasite $\mathrm{NaX}$ zeolites are synthesized by the hydrothermal method, using as starting material the diatomite, as a precursor for the production of sodium silicate, and aluminum post-consumer, as a precursor of the aluminum solution. The zeolites were characterized by X-Ray Powder Diffraction, (XRD), Infrared Spectroscopy with Attenuation (ATR-IR) and Thermogravimetric analysis (TGA), Differential Scanning Calorimetry (DSC) and Scanning Electronic Micoscopy (SEM). Sintered zeolites had a binding capacity of 4.8 $\mathrm{mmol} / \mathrm{g}$ of $\mathrm{Cu}^{2+}, 3.4 \mathrm{mmol} / \mathrm{g}$ for $\mathrm{Cd}^{2+}$ and $2.3 \mathrm{mmol} / \mathrm{g}$ of $\mathrm{Ni}^{2+}$, starting from the nitrates of these cations, which demonstrates the potential of these materials for the removal of heavy metals.
\end{abstract}

Key words: Diatomite, post-consumer aluminum; hydrothermal treatment; LTA-Na and Faujasite-NaX zeolites; Copper, Nickel and Cadmium

\section{Introducción}

Uno de los problemas más serios que enfrenta la industria en general es la generación de descargas residuales derivadas de los procesos industriales, cuya composición varía con el tipo de industria y/ o proceso; por esta razón, los efluentes industriales pueden contener sustancias disueltas y en suspensión, dentro de las cuales se encuentran compuestos orgánicos que pueden ser biodegradables o no biodegradables, y/o compuestos inorgánicos (sales de amonio, fosfatos, metales pesados, etc.), así como compuestos tóxicos (Nurul, et al., 2011); haciendo de este hecho un fenómeno reconocido como uno de los problemas de mayor responsabilidad ambiental que enfrenta la industria en Colombia.

Las zeolitas son aluminosilicatos con estructura cristalina, que contiene cavidades en forma de canales, ocupadas por grandes iones y moléculas de agua, ambos con libertad de movimiento permitiendo el intercambio iónico y la

\section{*Correspondencia:}

José A. Henao, jahenao@gmail.co

Recibido: 6 de mayo de 2014

Aceptado: 4 de agosto de 2014 
deshidratación reversible (Salam, et al., 2013; Wang \& Peng, 2010, Qiu \& Zheng, 2009). Su estructura consiste en una red tridimensional de tetraedros, con átomos de aluminio o silicio en el centro y átomos de oxigeno en los vértices. Las diferentes formas de coordinación de estos tetraedros, así como la relación silicio/aluminio originan diferentes tipos de zeolitas (Gómez, et al., 2001); a su vez, esta relación influye directamente sobre la tendencia hidrofílica o hidrofóbica del material, la cual se define en función de la densidad de átomos de aluminio en el interior de las cavidades de su estructura, en general la hidrofobicidad aumenta con un aumento en la relación $\mathrm{Si} / \mathrm{Al}$ (Herance et al., 2005). Cada átomo de aluminio que sustituye un átomo de silicio, proporciona una carga negativa, la cual se neutraliza con las cargas positivas que aportan los iones intercambiables, los cuales se unen a la estructura de la red mediante un enlace iónico que permite su intercambio por otro catión orgánico o inorgánico sin que la estructura cristalina de la zeolita sea alterada (Danil, et al., 2012; Kumar, et al., 2008; Herance, et al., 2005).

Pueden encontrarse en depósitos naturales, generalmente asociados con la activación hidrotérmica de rocas volcánicas vidriosas ó pueden ser sintetizadas con una gran variedad de materiales con alto contenido de silicio y aluminio. Como consecuencia de sus propiedades fisicoquímicas, son utilizadas en una amplia gama de aplicaciones industriales. Particularmente, la gran capacidad de intercambio catiónico le confiere a las zeolitas una alta aplicabilidad como intercambiadores iónicos en el tratamiento de aguas para la eliminación de amonio, fosfatos o metales pesados principalmente (Danil, et al., 2012, Borai, et al., 2009; Kumar, et al., 2008). Además, debido a su estructura de canales abiertos y vacíos, pueden ser usadas como tamices moleculares en la adsorción física de moléculas gaseosas y como catalizadores gracias a la presencia de protones como cationes intercambiables que pueden convertir a la zeolita en sólidos de elevada acidez (Herance, et al., 2005; Villavicencio et al., 2011).

Aunque actualmente gran parte del volumen total de la producción mundial de zeolitas es destinado para su uso en la formulación de detergentes (Wang \& Peng, 2010), se han definido otras áreas de aplicabilidad como la agricultura, la horticultura, materiales para construcción y el tratamiento de efluentes mineros y metalúrgicos entre otros, por lo que la síntesis de estos materiales a partir de materiales post-consumo o de fácil acceso facilita su aplicación en la industria (Zhang et al., 2013; Wang \& Peng, 2010; Salam et al., 2013).

Las diatomitas son rocas silícicas de origen biogénico, inertes y de baja toxicidad, compuestas por caparazones fosilizados de plantas acuáticas unicelulares morfológicamente sencillas, las diatomeas. Se forman por la acumulación sedimentaria de frústulas de diatomeas (Insung et al., 1999; Mohamed, 2010; Yi-Qiu, et al., 2012). Están constituidas esencialmente de sílice hidratada, ya sea en forma amorfa o cristalina según la concentración de sílice en la fuente de agua y la edad del yacimiento. Soluciones sobresaturadas de sílice favorecen la precipitación de ópalo el cual según la antigüedad del depósito cambia su morfología hasta convertirse en cuarzo (Pozo M., \& López M.J., 2004). Entre sus impurezas se encuentra la alúmina y el hierro, así como detritos sedimentarios que acompañan al material por su origen (Bahramian et al., 2008; Hadjar et al., 2008; Mohamedbakr H., \& Burkitbaev, M., 2009).

Por sus propiedades físicas, químicas y térmicas, como gran área superficial por unidad de masa, baja densidad aparente, químicamente inerte y aislante térmico en un amplio rango de temperatura; son aptas para diversas aplicaciones industriales, entre las que se encuentran su uso como filtro, absorbente y su uso como materia prima para la fabricación de ladrillos y cemento (Mohamed, 2010; Yin-qiu et al., 2012, Gerengi et al., 2013). No obstante, ha sido utilizada también como materia prima para la síntesis de nuevos materiales en aplicaciones más específicas como las zeolitas (López et al., 2005; Yang et al., 2011; Liu et al., 2014).

En este orden de ideas, la diatomita constituye una valiosa herramienta ya que gracias a sus propiedades fisicoquímicas, abre la posibilidad para el desarrollo de nuevas tecnologías sobre síntesis de materiales como las zeolitas, aluminosilicatos de estructura cristalina con gran capacidad de adsorción e intercambio iónico, de sales inorgánicas con metales pesados, para su aplicación en la remediación de problemas ambientales en el país, que hasta ahora no figuran dentro de los usos convencionales de la diatomita (Wajima T. \& Munakata K., 2012).

Adicionalmente, este trabajo permite potenciar su uso en nuevas aplicaciones y por lo tanto aumentar el valor agregado a los recursos de explotación industrial de diatomita en Colombia, promoviendo dentro del contexto de la minería de minerales no metálicos el desarrollo sustentable, la mitigación del impacto ambiental, el mejoramiento de ecosistemas y de la calidad de vida.

\section{Protocolo experimental}

La diatomita utilizada en el presente trabajo fue recolectada en la Vereda de Siatoca del Municipio de Chivata (Boyacá) a la altura del $\mathrm{km} 7$ al margen derecho de la vía principal que comunica Tunja con Toca.

\subsection{Reactivos}

Ácido clorhídrico 37\% (MERCK); Peróxido de hidrogeno 30\% (MERCK); Hidróxido de sodio 99\% (MERCK); Nitrato de cobre tri-hidratado (MERCK, extrapuro); Nitrato de Níquel hexa-hidratado (MERCK, Extrapuro), Nitrato de Cadmio marca tetra-hidratado (SIGMA-ALDRICH, 99\%) y Ácido Sulfúrico 98\% (MERCK). 


\subsection{Solubilización del silicio a partir de la diatomita}

Las muestras de diatomita fueron reducidas a polvo en un mortero de ágata, secadas al aire y tamizadas a través de una malla de $38 \mu \mathrm{m}$, para aumentar el área de contacto. La diatomita fue caracterizada superficialmente por Microscopia Electrónica de Barrido (MEB) (FEI QUANTA 200, con un alcance hasta 260.000 aumentos); para complementar la información de la MEB se tomaron Micrografías en un Microscopio Óptico convencional (Olympus, BX51), las Micrografías se tomaron con luz paralela con objetivos de 60 y 100x (este último de inmersión); la caracterización elemental se efectuó mediante Fluorescencia de Rayos-X (FRX) de longitud de onda dispersiva de $4 \mathrm{KW}$ utilizando un equipo marca BRUKER, modelo S8 TIGER; el estudio de la composición de fases cristalinas (programa especializado SEARCH/MATCH) y el cálculo del porcentaje de las fases cristalinas y material amorfo (programa especializado RIQAS) se realizó mediante Difracción de Rayos-X de muestras policristalinas (DRX) utilizando un equipo BRUKER, modelo D8 ADVANCE con geometría DA VINCI; la caracterización de los grupos funcionales se realizó mediante Espectroscopía Infrarroja con atenuación total (IRATR), utilizando un equipo BRUKER, modelo TENSOR 27, para determinar la Reflectancia Difusa de la muestras, se utilizó un espectrómetro marca SHIMADZU, modelo Tensor 27; la estabilidad térmica se estudió en un Calorímetro de Barrido Diferencial (CBD) calorímetro marca TA INSTRUMENTS, modelo DSCQ10 y la cuantificación de los lixiviados se realizó por Absorción Atómica (AA), utilizando un equipo PERKIN ELMER modelo 372.

\subsection{Calcinación}

La eliminación de los compuestos orgánicos presentes en la diatomita natural se realizó mediante oxidación térmica a $600^{\circ} \mathrm{C}$ durante 5 horas.

\subsection{Lixiviación}

El proceso de lixiviación se realizó a 15 muestras de $2 \mathrm{~g}$ de diatomita las cuales fueron sometidas a digestiones (temperatura de $90^{\circ} \mathrm{C}, 750 \mathrm{rpm}$ por $6 \mathrm{~h}$ en un autoclave de teflón de $65 \mathrm{~mL}$ de volumen) con $40 \mathrm{~mL}$ de cada una de las soluciones: $\mathrm{HCl} 4 \%-\mathrm{H}_{2} \mathrm{O}_{2} 2 \% \mathrm{p} / \mathrm{v}, \mathrm{H}_{2} \mathrm{SO}_{4} 2 \% \mathrm{p} / \mathrm{v}$ y $\mathrm{HNO}_{3} 2 \% \mathrm{p} / \mathrm{v}$. Las digestiones se aplicaron en la secuencia descrita en la Tabla 1. Una vez finalizado el proceso de lixiviación, los filtrados se analizaron por absorción atómica para determinar la concentración de hierro y aluminio extraídos.

\subsection{Extracción del silicio como silicato de sodio}

El sólido remanente de la lixiviación que presentó mayor concentración de silicio, se sometió a tratamiento hidrotérmico con una solución $3 \mathrm{M}$ de $\mathrm{NaOH}$ conservando la relación de $4 \mathrm{~g}$ de sólido por cada $40 \mathrm{~mL}$ de base, mezclados en un autoclave de teflón a $90^{\circ} \mathrm{C}, 750 \mathrm{rpm}$ por $6 \mathrm{~h}$ (Hollman et al., 1999). La cantidad de silicio extraído se determinó por AA.
Tabla 1. Combinación de las soluciones lixiviantes. Los números arábigos indican la secuencia utilizada.

\begin{tabular}{|c|c|}
\hline Número & Secuencia de las digestiones \\
\hline 1 & 1. $\mathrm{HCl} 4 \% \mathrm{p} / \mathrm{v}-\mathrm{H}_{2} \mathrm{O}_{2} 2 \% \mathrm{p} / \mathrm{v}$ \\
\hline 2 & 1. $\mathrm{HCl} 4 \% \mathrm{p} / \mathrm{v}-\mathrm{H}_{2} \mathrm{O}_{2} 2 \% \mathrm{p} / \mathrm{v} ; 2 . \mathrm{HNO}_{3} 2 \% \mathrm{p} / \mathrm{v}$ \\
\hline 3 & 1. $\mathrm{HCl} 4 \% \mathrm{p} / \mathrm{v}-\mathrm{H}_{2} \mathrm{O}_{2} 2 \% \mathrm{p} / \mathrm{v} ; 2$. $\mathrm{HNO}_{3} 2 \% \mathrm{p} / \mathrm{v} ; 3 . \mathrm{H}_{2} \mathrm{SO}_{4} 2 \% \mathrm{p} / \mathrm{v}$ \\
\hline 4 & 1. $\mathrm{H}_{2} \mathrm{SO}_{4} 2 \% \mathrm{p} / \mathrm{v}$ \\
\hline 5 & 1. $\mathrm{H}_{2} \mathrm{SO}_{4} 2 \% \mathrm{p} / \mathrm{v} ; 2 . \mathrm{HNO}_{3} 2 \% \mathrm{p} / \mathrm{v}$ \\
\hline 6 & 1. $\mathrm{HNO}_{3} 2 \% \mathrm{p} / \mathrm{v}$ \\
\hline 7 & 1. $\mathrm{HNO}_{3} 2 \% \mathrm{p} / \mathrm{v} ; 2$. $\mathrm{HCl} 4 \% \mathrm{p} / \mathrm{v}-\mathrm{H}_{2} \mathrm{O}_{2} 2 \% \mathrm{p} / \mathrm{v}$ \\
\hline 8 & 1. $\mathrm{HCl} 4 \% \mathrm{p} / \mathrm{v}-\mathrm{H}_{2} \mathrm{O}_{2} 2 \% \mathrm{p} / \mathrm{v} ; 2 . \mathrm{H}_{2} \mathrm{SO}_{4} 2 \% \mathrm{p} / \mathrm{v}$ \\
\hline 9 & 1. $\mathrm{HNO}_{3} 2 \% \mathrm{p} / \mathrm{v} ; 2 . \mathrm{H}_{2} \mathrm{SO}_{4} 2 \% \mathrm{p} / \mathrm{v}$ \\
\hline 10 & 1. $\mathrm{H}_{2} \mathrm{SO}_{4} 2 \% \mathrm{p} / \mathrm{v} ; 2 . \mathrm{HCl} 4 \% \mathrm{p} / \mathrm{v}-\mathrm{H}_{2} \mathrm{O}_{2} 2 \% \mathrm{p} / \mathrm{v}$ \\
\hline 11 & 1. $\mathrm{HNO}_{3} 2 \% \mathrm{p} / \mathrm{v} ; 2 . \mathrm{H}_{2} \mathrm{SO}_{4} 2 \% \mathrm{p} / \mathrm{v} ; 3 . \mathrm{HCl} 4 \% \mathrm{p} / \mathrm{v}-\mathrm{H}_{2} \mathrm{O}_{2} 2 \% \mathrm{p} / \mathrm{v}$ \\
\hline 12 & 1. $\mathrm{H}_{2} \mathrm{SO}_{4} 2 \% \mathrm{p} / \mathrm{v} ; \mathbf{2}$. $\mathrm{HCl} 4 \% \mathrm{p} / \mathrm{v}-\mathrm{H}_{2} \mathrm{O}_{2} 2 \% \mathrm{p} / \mathrm{v} ; \mathbf{3} . \mathrm{HNO}_{3} 2 \% \mathrm{p} / \mathrm{v}$ \\
\hline 13 & 1. $\mathrm{H}_{2} \mathrm{SO}_{4} 2 \% \mathrm{p} / \mathrm{v} ; 2 . \mathrm{HNO}_{3} 2 \% \mathrm{p} / \mathrm{v} ; 3 . \mathrm{HCl} 4 \% \mathrm{p} / \mathrm{v}-\mathrm{H}_{2} \mathrm{O}_{2} 2 \% \mathrm{p} / \mathrm{v}$ \\
\hline 14 & 1. $\mathrm{HCl} 4 \% \mathrm{p} / \mathrm{v}-\mathrm{H}_{2} \mathrm{O}_{2} 2 \% \mathrm{p} / \mathrm{v} ; 2 . \mathrm{H}_{2} \mathrm{SO}_{4} 2 \% \mathrm{p} / \mathrm{v} ; 3 . \mathrm{HNO}_{3} 2 \% \mathrm{p} / \mathrm{v}$ \\
\hline 15 & 1. $\mathrm{HNO}_{3} 2 \% \mathrm{p} / \mathrm{v} ; \mathbf{2} . \mathrm{HCl} 4 \% \mathrm{p} / \mathrm{v}-\mathrm{H}_{2} \mathrm{O}_{2} 2 \% \mathrm{p} / \mathrm{v} ; 3$. $\mathrm{H}_{2} \mathrm{SO}_{4} 2 \% \mathrm{p} / \mathrm{v}$ \\
\hline
\end{tabular}

\subsection{Extracción del aluminio de las anillas de cerveza.}

Las anillas de aluminio se calcinaron a $600^{\circ} \mathrm{C}$ por $3 \mathrm{~h}$, posteriormente el material se sometió a tratamiento alcalino con $\mathrm{NaOH} 3 \mathrm{M}$ durante $2 \mathrm{~h}$, con agitación constante y conservando la estequiometria propuesta en la Reacción 1, (Aleksandrov et al. 2003). La solución se caracterizó por AA.

$2 \mathrm{Al}(\mathrm{s})+2 \mathrm{NaOH}(\mathrm{ac})+6 \mathrm{H}_{2} \mathrm{O}(\mathrm{l}) \rightleftharpoons 2 \mathrm{Na}\left[\mathrm{Al}(\mathrm{OH})_{4}\right](\mathrm{ac})+3 \mathrm{H}_{2}(\mathrm{~g})$

Reacción 1 Extracción de Aluminio

\subsection{Síntesis y Caracterización de zeolitas.}

\section{Síntesis de la Zeolita LTA-Na.}

Partiendo de las soluciones de silicio y aluminio disuelto, se tomaron tres autoclaves de teflón de $65 \mathrm{~mL}$ y a cada uno se le mezclaron los precursores gota a gota con agitación constante conservando una relación $\mathrm{Si} / \mathrm{Al}$ de 1 hasta completar $40 \mathrm{~mL}$ de la mezcla. Al finalizar la adición, los autoclaves con los crudos de reacción se sometieron a envejecimiento a $90^{\circ} \mathrm{C}$, el primero por $3 \mathrm{~h}$, el segundo por $6 \mathrm{~h}$ y tercero por $9 \mathrm{~h}$, al término de este tiempo los materiales se filtraron y los precipitados se lavaron con agua tipo 1 , hasta lograr un $\mathrm{pH}=7$ en las aguas de lavado. Los sólidos se secaron a $90^{\circ} \mathrm{C}$ durante $10 \mathrm{~h}$, se denominaron como LTA-3, LTA-6 y LTA-9 y fueron caracterizados por DRXP, ATR-IR, ATG y CBD.

\section{Síntesis de la Zeolita FAUJASITA-NaX.}

Los precursores de aluminio y silicio se mezclaron en 16 autoclaves de teflón de $65 \mathrm{~mL}$, hasta completar un volumen de $40 \mathrm{~mL}$ de la mezcla conservando una relación de $\mathrm{Si} / \mathrm{Al}$ de 1,2 para los ocho primeros y de 2,4 para los ocho restantes. Al 
término de cada una de las adiciones, el crudo de reacción se dejo en agitación constate por 30min. Posteriormente, los 16 crudos de reacción se sometieron a un estudio de maduración como se muestra a continuación.

\section{Estudio del tiempo de maduración.}

Los crudos de reacción mezclados bajo la relación $\mathrm{Si} / \mathrm{Al}$ de 1,2 , se sometieron a maduración de 24 y $120 \mathrm{~h}$ a temperatura ambiente. Una vez finalizada la maduración, los crudos de reacción se sometieron a envejecimiento a $90^{\circ} \mathrm{C}$ por $0,8,16$ y $24 \mathrm{~h}$. Para los crudos de reacción mezclados en una relación $\mathrm{Si} / \mathrm{Al} 2,4$, se les realizó un proceso de maduración a 24 y 48 horas. Posteriormente, se sometieron a envejecimiento por 0 , 8,16 y $24 \mathrm{~h}$. Cada uno de los productos obtenidos luego del envejecimiento, se filtraron, se lavaron con agua desionizada y se secaron a $90^{\circ} \mathrm{C}$. Los sólidos se denominaron DE1, DE2, DE3 y DE4 para la relación Si/Al 1,2 y maduración de 24h siguiendo la secuencia de los tiempos de envejecimiento; mientras que para la maduración $120 \mathrm{~h}$ se nombraron, DE5, DE6, DE7 y DE8. Los sólidos con relación 2,4 y 24h de maduración se denominaron ADE1, ADE2, ADE3 y ADE4 y los de 48 horas de maduración ADE5, ADE6, ADE7 y ADE8. Todos los materiales sintetizados se caracterizaron por DRXP.

\subsection{Pruebas de intercambio iónico}

Para las pruebas de intercambio iónico se prepararon tres soluciones de 2L de Níquel, Cobre y Cadmio a 1000mg/L, partiendo de nitrato de níquel, nitrato de cobre y nitrato de cadmio.

\section{Intercambio catiónico de la zeolita LTA-Na.}

Se tomaron tres muestras de $0,1000 \mathrm{~g}$ de la muestra de zeolita sintetizada LTA-3, se sometieron a un tratamiento de secado a $300^{\circ} \mathrm{C}$ por $3 \mathrm{~h}$ en atmosfera de argón. Transcurrido este tiempo, el material se llevó a $50^{\circ} \mathrm{C}$, luego, se mezcló con $26,9 \mathrm{~mL}$ de cada uno de los nitratos de níquel, cobre y cadmio, para luego tomar una alícuota de $300 \mu \mathrm{L}$ de la solución cada 30 minutos hasta completar $2 \mathrm{~h}$ y 30 minutos. Cada una de las alícuotas se analizó por AA para cada metal $(\mathrm{Cu}, \mathrm{Ni}$ y $\mathrm{Cd})$. El procedimiento anterior se repitió para las muestras LTA-6 y LTA-9.

\section{Intercambio de la zeolita FAUJASITA NaX.}

Se tomaron tres muestras de $0,1000 \mathrm{~g}$ de la muestra de zeolita sintetizada ADE1, la zeolita se sometió a un tratamiento de secado a $300^{\circ} \mathrm{C}$ por $3 \mathrm{~h}$ en atmosfera de argón. Transcurrido este tiempo, el material se llevó a $50^{\circ} \mathrm{C}$ y luego se mezcló con $39,9 \mathrm{~mL}$ de cada uno de los nitratos de níquel, cobre y cadmio. Finalmente, se tomaron alícuotas de $300 \mu \mathrm{L}$ de la solución cada 30 minutos hasta completar $2 \mathrm{~h}$ y 30 minutos. Cada una de las alícuotas se analizó por AA para cada metal $(\mathrm{Cu}, \mathrm{Ni}$ y $\mathrm{Cd})$. El procedimiento anterior se repitió para las muestras ADE2, ADE3 y ADE4.

\section{RESULTADOS Y DISCUSIÓN}

\subsection{Caracterización de la diatomita}

\section{Microscopia Electrónica de Barrido}

Mediante Microscopía Electrónica de Barrido se encontró que la especie de diatomeas más abundante en la muestra, pertenece a la clase de diatomeas céntricas del orden Aulacoseirales, más específicamente la especie Aulacoseira granulata. En la Figura 1a se puede apreciar la forma cilíndrica y porosa de la frústula, y en la Figura 1b se observa que poseen valvas circulares, las cuales se unen mediante espinas que se encuentran en el borde (Naranjo et al., 2007; Oliva et al., 2005).

Aunque en menor proporción, se estableció que en la muestra objeto de estudio hay presencia de otros dos tipos de diatomeas pertenecientes a la clase de diatomeas penadas con rafe, más específicamente las especies Navicula Radiosa y Pinularia of (Naranjo et al., 2007; Oliva et al., 2005), como se puede apreciar en la Figura 2.
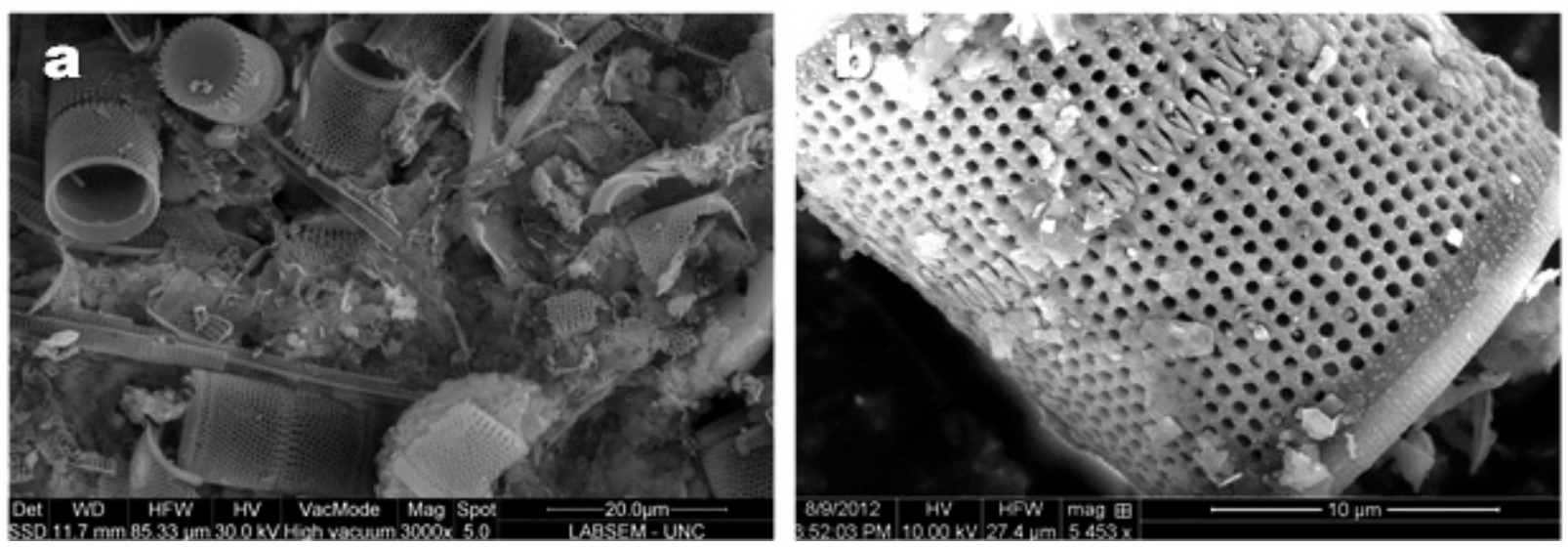

Figura 1. Imágenes por microscopia electrónica de barrido de la diatomita. 

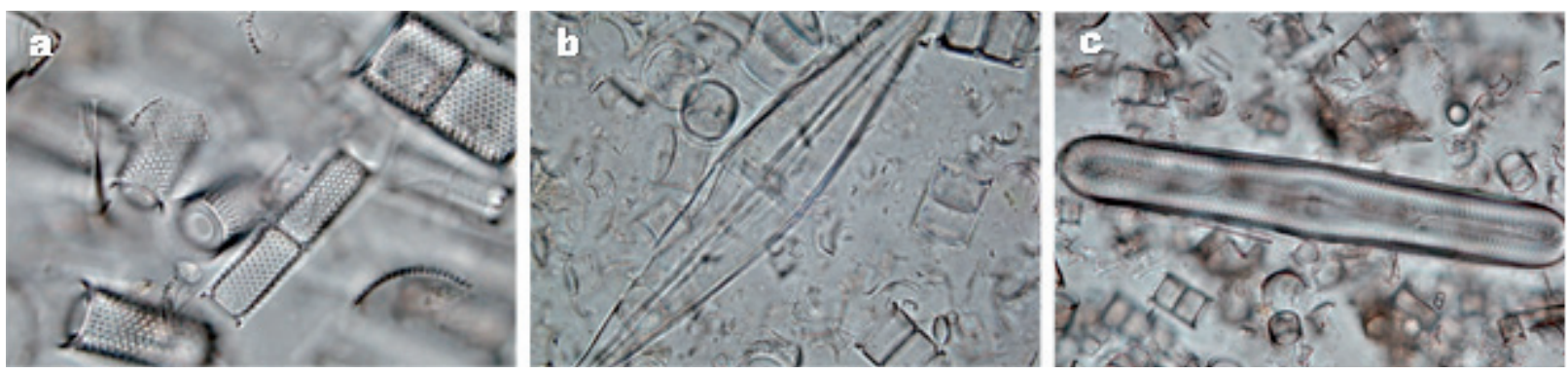

Figura 2. Diferentes especies de diatomeas fósiles identificadas. a) Aulacoseira granulata, b) Navicula Radiosa, c) Pinularia cf.

\section{Análisis Elemental}

A partir del análisis elemental por fluorescencia de rayos $\mathrm{X}$ de la diatomita, fue posible conocer la composición del material expresada en porcentaje en peso, indicando que sus componentes mayoritarios, son silicio, aluminio e hierro, los cuales presentados como óxidos están del orden de 71,853, 21,264 y $3,191 \%$ en peso, respectivamente. Además, se evidencia la presencia de los elementos potasio, titanio, calcio y magnesio entre otros, en menores proporciones. De acuerdo con estos porcentajes, la diatomita se clasifica como un material puzolánico de clase $\mathrm{N}$ según la norma ASTM C618, ya que la suma de los porcentajes de sus componentes mayoritarios $\left(\mathrm{SiO}_{2}, \mathrm{Al}_{2} \mathrm{O}_{3}\right.$ y $\left.\mathrm{Fe}_{2} \mathrm{O}_{3}\right)$ supera el $70 \%$, además, su contenido de azufre es inferior al establecido por la norma el cual debe ser menor al $4 \%$.

$\mathrm{El}$ alto contenido silicio (expresado como $\mathrm{SiO}_{2}$ ), aproximadamente del $71,853 \%$ en peso del material, hace evidente el potencial de la tierra de diatomáceas como fuente de silicio para su uso como precursor en la síntesis de materiales zeolíticos, ya sea en su forma natural ó modificada después de la extracción de cationes presentes en la misma (Liu et al, 2014; Wajima T. \& Munakata K., 2012; Shan et al., 2004).

\section{Caracterización Mineralógica de la diatomita}

\section{Análisis Cualitativo}

Mediante el análisis cualitativo de fases cristalinas, se estableció la presencia de las fases Moscovita PDF 85-2147, Caolinita PDF 78-1996, Cuarzo PDF 85-0794, Anatasa 841285 y Vermiculita 76-0847. Además, se destaca la presencia de una elevación del ruido de fondo en la región de 2,9 a

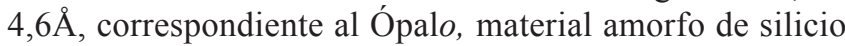
presente en la muestra (Naranjo et al., 2007). El resultado del análisis cualitativo se presenta en la Figura 3. A partir del anterior resultado, se puede inferir que todas las fases cristalinas presentes diferentes de la Anatasa, hacen parte de la fuente de silicio en el material, además que el aporte de aluminio en la diatomita, es dado principalmente por la Caolinita y la Vermiculita.

\section{Análisis cuantitativo}

A partir de la cuantificación de las fases cristalinas en la muestra, se evidenció la presencia de una cantidad significativa de cuarzo, muy posiblemente debido a que la fuente de agua del yacimiento de diatomita tenía baja concentración de sílice o quizás por causa de fenómenos de envejecimiento del ópalo (Alonso-Zarza, 2010). Los resultados obtenidos del refinamiento del perfil de difracción por mínimos cuadrados empleando el Software comercial RIQAS, usando como patrón el Corindón PDF 73-1512, muestran que la diatomita presenta 40,1\% de material amorfo, 22,7\% de Muscovita, $20,1 \%$ de Caolinita, $15,8 \%$ de Cuarzo y $1,20 \%$ de Vermiculita. El contenido de Anatasa en la diatomita no es cuantificable, debido a la baja proporción en la que se encuentra dentro del material, por lo que se reporta dentro del material amorfo. Aunque según el análisis cuantitativo, el $40,1 \%$ de la muestra de diatomita objeto de estudio corresponde a material amorfo, esto no necesariamente indica una falta de ordenamiento interno de parte de los átomos que conforman dicha estructura, muy probablemente el tamaño de los cristales de estos sólidos es muy pequeño y no son detectables por Difracción de Rayos-X de Muestras policristalinas.

\subsection{Tratamientos previos a la síntesis de la zeolita}

\section{Calcinación}

Con la incineración de la muestra, se lograron eliminar los compuestos orgánicos volátiles presentes en la diatomita y parte del agua de constitución de los minerales presentes en el material, correspondientes a aproximadamente el $9 \%$ del peso inicial de la muestra sólida. Se puede inferir que después de la calcinación, la diatomita ha sufrido transformaciones cristalográficas en donde su estructura pierde iones hidroxilo (Ghosh et al., 2004) y pasa de un estado cristalino a un estado amorfo, en el cual sus componentes son más reactivos (Chaisena and Rangsriwatananon, 2004; Rodrígez et al., 2005; Yilmaz and Ediz, 2008), debido a la falta de ordenamiento interno de los átomos constituyentes de cada mineral. 


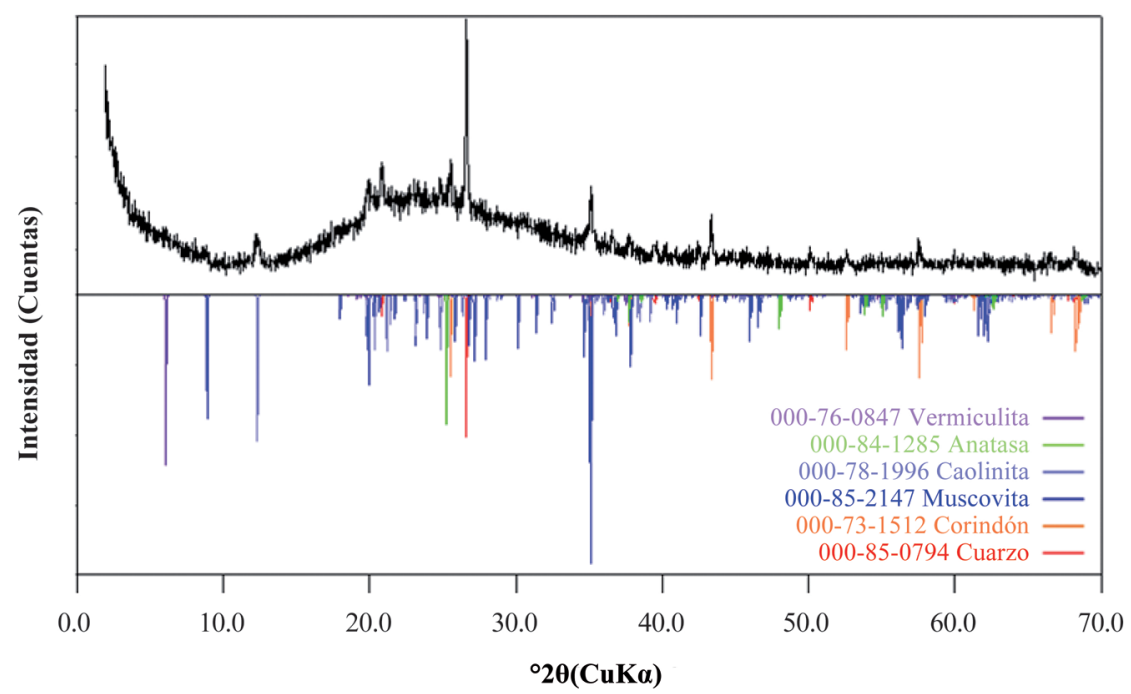

Figura 3. Perfil de difracción de rayos X de la diatomita.

\section{Lixiviación}

Después de realizado el tratamiento ácido bajo las condiciones anteriormente expuestas, fue posible reducir gran parte de los cationes presentes en la muestra de diatomita, diferentes al silicio. En la Tabla 2 se muestran los resultados de AA para aluminio e hierro en los filtrados obtenidos tras cada uno de los procesos de digestión. Todos los filtrados se redujeron hasta un volumen de $19 \mathrm{~mL}$. De lo expuesto en la Tabla 2 se puede evidenciar que la extracción de la secuencia 3 , es la que mayor cantidad de aluminio retira (9099,0ppm), mientras que para el caso del hierro (2082,6ppm) es la secuencia 13 la que elimina la mayor proporción. Además, de las tres soluciones ácidas la digestión más efectiva para retirar los iones de hierro y aluminio es la de $4 \% \mathrm{HCl}-2 \% \mathrm{H}_{2} \mathrm{O}_{2} \mathrm{p} / \mathrm{v}$. Lo anterior se debe a la alta dureza que presenta el cloruro, comparada con la del nitrato y el sulfato, los cuales al tener su carga distribuida entre tres y cuatro oxígenos respectivamente, se hacen más polarizables, y como los cationes de Fe (II, III) y el Al(III) presentan una dureza media comparada con la del $\mathrm{Si}(\mathrm{IV})$, reaccionan para formar sales que tienden a ser solubles al constituir una reacción catión-anión del tipo Blando-Duro (Bernal J.P. \& Railsback L.,2008).

\section{Extracción del silicio como silicato de sodio}

Para extraer el silicato, se tomó el sólido tratado con la secuencia 3, debido a que el aluminio es el mayor contaminante presente en la diatomita natural, la cual, al aplicar la secuencia 3 partiendo de $4,000 \mathrm{~g}$ de diatomita calcinada, se llega a $3,109 \mathrm{~g}$ de diatomita tratada, lo que indica que con las lixiviaciones el material presenta una reducción de masa del 22,28\% con la composición descrita en la Tabla 3 lo anterior permite establecer que el porcentaje de extracción del $\mathrm{Al}_{2} \mathrm{O}_{3}$ y $\mathrm{Fe}_{2} \mathrm{O}_{3}$ contaminantes mayoritarios) es del 57,937 y $70,844 \%$ respectivamente, lo cual se traduce en una concentración del $\mathrm{SiO}_{2}$ en la diatomita modificada del $83,812 \%$, logrando aumentar la concentración en el material un 11,959\% empleando soluciones diluidas de ácidos, este resultado es mejor, que el obtenido por (Rangsriwatananon et al, 2008), en los que se logra una concentración de $\mathrm{SiO}_{2}$ en un diatomita modificada del $22,52 \%$, pero empleando una solución de ácido sulfúrico del $58,8 \%$ p/v $(6 \mathrm{M})$ por $24 \mathrm{~h}$ a $100^{\circ} \mathrm{C}$ y presión autógena, mientras que en el presente trabajo se logran resultados semejantes empleando soluciones diluidas (1. $\mathrm{HCl} 4 \% \mathrm{p} / \mathrm{v}-\mathrm{H}_{2} \mathrm{O}_{2} 2 \% \mathrm{p} / \mathrm{v} ; 2$. $\mathrm{HNO}_{3} 2 \% \mathrm{p} / \mathrm{v} ; 3$. $\mathrm{H}_{2} \mathrm{SO}_{4} 2 \% \mathrm{p} / \mathrm{v}$ ) en un término de $18 \mathrm{~h}$ a $90^{\circ} \mathrm{C}$, lo que hace el proceso de tratamiento más económico y ambientalmente más beneficioso, teniendo en cuenta la dificultad para sintetizar y obtener el $\mathrm{H}_{2} \mathrm{SO}_{4}$ a nivel industrial (Davenport et al, 2006).

Después de someter los $4,000 \mathrm{~g}$ de diatomita modifica al tratamiento alcalino se obtuvo $1,000 \mathrm{~g}$ de sólido con la composición descrita en la columna 5 de la Tabla 3 , resultados que permiten afirmar que el porcentaje de solubilización del $\mathrm{SiO}_{2}$ fue del 90,004\%, además se evidenció la fijación de sodio en la muestra (en un 10401,25\%), esto indica que en el material sólido el $\mathrm{SiO}_{2}$ y el $\mathrm{Al}_{2} \mathrm{O}_{3}$ que no se solubiliza, reacciona con la base adicionada generando aluminosilicatos, proceso que es usual en el tratamiento alcalino de minerales (Liu et al, 2004), tal como lo confirma el análisis cualitativo de DRX, en el que se evidenció la presencia del aluminosilicato Zeolita Thetha-1 PDF-37-357. Otras fases encontradas fueron la Illita $-2 M$ PDF-26-911, Oxido de titanio (TiO 2) PDF-77-443 y Oxido de silicio PDF-77-1060 ( $\mathrm{SiO}_{2}$ ). Por otro lado, la concentración de la solución de silicato a partir de la diatomita modificada fue de 24017,60 ppm de Si. 
Tabla 2. Resultados de AA de los filtrados generados en la lixiviación de la diatomita natural.

\begin{tabular}{|c|c|c|c|}
\hline Nùmero & Soluciones Extractoras & $\begin{array}{l}\text { Concentración Hierro } \\
\text { en } 19 \mathrm{~mL} \pm 0,1 \mathrm{ppm}\end{array}$ & $\begin{array}{l}\text { Concentración Aluminio } \\
\text { en } 19 \mathrm{~mL} \pm 0,2 \mathrm{ppm}\end{array}$ \\
\hline 1 & 1. $\mathrm{HCl} 4 \% \mathrm{p} / \mathrm{v}-\mathrm{H}_{2} \mathrm{O}_{2} 2 \% \mathrm{p} / \mathrm{v}$ & 1027,0 & 3867,0 \\
\hline 2 & 1. $\mathrm{HCl} 4 \% \mathrm{p} / \mathrm{v}-\mathrm{H}_{2} \mathrm{O}_{2} 2 \% \mathrm{p} / \mathrm{v} ; 2 . \mathrm{HNO}_{3} 2 \% \mathrm{p} / \mathrm{v}$ & 1052,0 & 4012,0 \\
\hline 3 & 1. $\mathrm{HCl} 4 \% \mathrm{p} / \mathrm{v}-\mathrm{H}_{2} \mathrm{O}_{2} 2 \% \mathrm{p} / \mathrm{v} ; 2 . \mathrm{HNO}_{3} 2 \% \mathrm{p} / \mathrm{v} ; 3 . \mathrm{H}_{2} \mathrm{SO}_{4} 2 \% \mathrm{p} / \mathrm{v}$ & 1074,0 & 9099,0 \\
\hline 4 & 1. $\mathrm{H}_{2} \mathrm{SO}_{4} 2 \% \mathrm{p} / \mathrm{v}$ & 427,0 & 2957,0 \\
\hline 5 & 1. $\mathrm{H}_{2} \mathrm{SO}_{4} 2 \% \mathrm{p} / \mathrm{v} ; 2 . \mathrm{HNO}_{3} 2 \% \mathrm{p} / \mathrm{v}$ & 1083,0 & 4094,0 \\
\hline 6 & 1. $\mathrm{HNO}_{3} 2 \% \mathrm{p} / \mathrm{v}$ & 60,5 & 3185,0 \\
\hline 7 & 1. $\mathrm{HNO}_{3} 2 \% \mathrm{p} / \mathrm{v} ; 2 . \mathrm{HCl} 4 \% \mathrm{p} / \mathrm{v}-\mathrm{H}_{2} \mathrm{O}_{2} 2 \% \mathrm{p} / \mathrm{v}$ & 1515,5 & 5915,0 \\
\hline 8 & 1. $\mathrm{HCl} 4 \% \mathrm{p} / \mathrm{v}-\mathrm{H}_{2} \mathrm{O}_{2} 2 \% \mathrm{p} / \mathrm{v} ; \mathbf{2} . \mathrm{H}_{2} \mathrm{SO}_{4} 2 \% \mathrm{p} / \mathrm{v}$ & 1512,4 & 4349,3 \\
\hline 9 & 1. $\mathrm{HNO}_{3} 2 \% \mathrm{p} / \mathrm{v} ; 2 . \mathrm{H}_{2} \mathrm{SO}_{4} 2 \% \mathrm{p} / \mathrm{v}$ & 688,3 & 6370,0 \\
\hline 10 & 1. $\mathrm{H}_{2} \mathrm{SO}_{4} 2 \% \mathrm{p} / \mathrm{v} ; 2 . \mathrm{HCl} 4 \% \mathrm{p} / \mathrm{v}-\mathrm{H}_{2} \mathrm{O}_{2} 2 \% \mathrm{p} / \mathrm{v}$ & 1625,3 & 5459,0 \\
\hline 11 & 1. $\mathrm{HNO}_{3} 2 \% \mathrm{p} / \mathrm{v} ; 2 . \mathrm{H}_{2} \mathrm{SO}_{4} 2 \% \mathrm{p} / \mathrm{v} ; 3 . \mathrm{HCl} 4 \% \mathrm{p} / \mathrm{v}-\mathrm{H}_{2} \mathrm{O}_{2} 2 \% \mathrm{p} / \mathrm{v}$ & 1715,3 & 7534,0 \\
\hline 12 & 1. $\mathrm{H}_{2} \mathrm{SO}_{4} 2 \% \mathrm{p} / \mathrm{v} ; 2 . \mathrm{HCl} 4 \% \mathrm{p} / \mathrm{v}-\mathrm{H}_{2} \mathrm{O}_{2} 2 \% \mathrm{p} / \mathrm{v} ; 3 . \mathrm{HNO}_{3} 2 \% \mathrm{p} / \mathrm{v}$ & 1690,0 & 5823,0 \\
\hline 13 & 1. $\mathrm{H}_{2} \mathrm{SO}_{4} 2 \% \mathrm{p} / \mathrm{v} ; 2 . \mathrm{HNO}_{3} 2 \% \mathrm{p} / \mathrm{v} ; 3 . \mathrm{HCl} 4 \% \mathrm{p} / \mathrm{v}-\mathrm{H}_{2} \mathrm{O}_{2} 2 \% \mathrm{p} / \mathrm{v}$ & 2082,6 & 4894,0 \\
\hline 14 & 1. $\mathrm{HCl} 4 \% \mathrm{p} / \mathrm{v}-\mathrm{H}_{2} \mathrm{O}_{2} 2 \% \mathrm{p} / \mathrm{v} ; 2 . \mathrm{H}_{2} \mathrm{SO}_{4} 2 \% \mathrm{p} / \mathrm{v} ; 3 . \mathrm{HNO}_{3} 2 \% \mathrm{p} / \mathrm{v}$ & 1560,0 & 4549,5 \\
\hline 15 & 1. $\mathrm{HNO}_{3} 2 \% \mathrm{p} / \mathrm{v} ; 2 . \mathrm{HCl} 4 \% \mathrm{p} / \mathrm{v}-\mathrm{H}_{2} \mathrm{O}_{2} 2 \% \mathrm{p} / \mathrm{v} ; 3 . \mathrm{H}_{2} \mathrm{SO}_{4} 2 \% \mathrm{p} / \mathrm{v}$ & 1569,1 & 6155,2 \\
\hline
\end{tabular}

Tabla 3. Análisis elemental por fluorescencia de rayos X (FRX) de la diatomita natural y modificada.

\begin{tabular}{|c|c|c|c|c|c|}
\hline \multirow[t]{3}{*}{ Compuesto } & \multirow{3}{*}{$\begin{array}{c}\text { Diatomita } \\
\text { Natural (DN) } \\
\text { Porcentaje (p/p) }\end{array}$} & \multirow{3}{*}{$\begin{array}{c}\text { Diatomita } \\
\text { Modificada (DM) } \\
\text { Porcentaje (p/p) }\end{array}$} & \multirow{2}{*}{$\begin{array}{c}\text { Porcentaje de } \\
\text { Extracción }(\mathbf{\% E}) \\
\% E=\left(1-\frac{3,11 g * \% D M}{4,00 g * \% D N}\right) * 100 \%\end{array}$} & \multirow{2}{*}{$\begin{array}{c}\text { Diatomita después } \\
\text { de la extracción con } \\
\text { NaOH 3M (DE) }\end{array}$} & \multirow{2}{*}{$\begin{array}{c}\begin{array}{c}\text { Porcentaje de } \\
\text { Solubilización }(\mathbf{0} \mathbf{S})\end{array} \\
\% S=\left(1-\frac{1,0 g * \% D E}{4,0 g * \% D M}\right) * 100 \%\end{array}$} \\
\hline & & & & & \\
\hline & & & Porcentaje (p/p) & Porcentaje (p/p) & Porcentaje (p/p) \\
\hline $\mathrm{SiO}_{2}$ & $71,853 \%$ & $83,812 \%$ & $9,339 \%$ & $33,374 \%$ & $90,004 \%$ \\
\hline $\mathrm{Al}_{2} \mathrm{O}_{3}$ & $21,264 \%$ & $11,504 \%$ & $57,937 \%$ & $27,376 \%$ & $40,508 \%$ \\
\hline $\mathrm{Fe}_{2} \mathrm{O}_{3}$ & $3,191 \%$ & $1,197 \%$ & $70,844 \%$ & $4,642 \%$ & $3,049 \%$ \\
\hline $\mathrm{K}_{2} \mathrm{O}$ & $1,471 \%$ & $1,322 \%$ & $41,251 \%$ & $5,287 \%$ & $0,019 \%$ \\
\hline $\mathrm{TiO}$ & $0,772 \%$ & $0,686 \%$ & $30,933 \%$ & $2,534 \%$ & $7,653 \%$ \\
\hline $\mathrm{CaO}$ & $0,483 \%$ & $0,000 \%$ & $100,000 \%$. & $0,000 \%$ & $0,000 \%$ \\
\hline $\mathrm{MgO}$ & $0,438 \%$ & $0,163 \%$ & $71,066 \%$ & $0,18 \%$ & $72,546 \%$ \\
\hline $\mathrm{BaO}$ & $0,080 \%$ & $0,060 \%$ & $41,688 \%$ & $0,12 \%$ & $50,000 \%$ \\
\hline $\mathrm{ZrO}_{2}$ & $0,017 \%$ & $0,014 \%$ & $35,970 \%$ & $0,031 \%$ & $44,643 \%$ \\
\hline $\mathrm{SO}_{3}$ & $0,148 \%$ & $0,066 \%$ & $65,328 \%$ & $0,040 \%$ & $84,848 \%$ \\
\hline $\mathrm{SrO}$ & $0,006 \%$ & $0,000 \%$ & $100,000 \%$. & $0,000 \%$ & $0,000 \%$ \\
\hline $\mathrm{P}_{2} \mathrm{O}_{5}$ & $0,170 \%$ & $0,040 \%$ & $81,706 \%$ & $0,000 \%$ & $100,000 \%$. \\
\hline $\mathrm{RbO}_{2}$ & $0,006 \%$ & $0,005 \%$ & $35,208 \%$ & $0,007 \%$ & $29,52 \%$ \\
\hline $\mathrm{Na}_{2} \mathrm{O}$ & $0,050 \%$ & $0,060 \%$ & $6,700 \%$ & $25,203 \%$ & $-10401,25 \%$, fijado $0,252 \mathrm{~g}$ \\
\hline $\mathrm{ZnO}$ & $0,010 \%$ & $0,000 \%$ & $100,000 \%$. & $0,000 \%$ & $0,000 \%$ \\
\hline $\mathrm{V}_{2} \mathrm{O}_{5}$ & $0,031 \%$ & $0,004 \%$ & $89,968 \%$ & $0,000 \%$ & $100,000 \%$. \\
\hline $\mathrm{MnO}$ & $0,010 \%$ & N.C & $100,00 \% \ldots$ & $0,000 \%$ & $0,000 \%$ \\
\hline $\mathrm{H}_{2} \mathrm{O}$ & $0,000 \%$ & $1,067 \%$ & Fijaron $0,033 \mathrm{~g}$ & $2,206 \%$ & Fijaron $0,023 \mathrm{~g}$ \\
\hline
\end{tabular}




\section{Pre-tratamiento hidrotérmico de laminas de aluminio}

Al tomar 20,000g de la muestra de anillas calcinadas y someterlas al tratamiento básico, se obtuvieron $12,730 \mathrm{~g}$ de una muestra grisácea, la cual al realizar el análisis por DRX no permitió identificar ninguna fase cristalina, su análisis elemental se muestra en la Tabla 4. lo anterior indica que el porcentaje de solubilización alcalina del aluminio es del $91,15 \%$, permitiendo obtener una solución con una concentración de aluminio de 58103,75ppm.

\section{Síntesis de la zeolita LTA.}

$\mathrm{Al}$ tomar tres autoclaves de $65 \mathrm{~mL}$ y agregarles a cada uno $35,73 \mathrm{~mL}$ de solución silicato de $24017,60 \mathrm{ppm}$ de silicio y $14,20 \mathrm{~mL}$ de solución de aluminato de $58103,75 \mathrm{ppm}$ de aluminio y realizando el estudio de tiempo de envejecimiento de 3,6 y 9 h se obtuvieron los perfiles de difracción de Rayos-X descritos en la Figura 4. A partir de los patrones de difracción se puede establecer la presencia de una fase cristalina correspondiente a la zeolita LTA-Na. Los espectros de IR-ATR para los tres productos presentaron frecuencias de vibración típicas de la zeolita LTA, las cuales están representadas por bandas de estiramientos Al-O localizadas en la región entre $1250-950 \mathrm{~cm}^{-1}$ y $770-630 \mathrm{~cm}^{-1}$, bandas en la región entre $650-500 \mathrm{~cm}^{-1}$ relacionadas con la presencia de dobles anillos en la estructura y bandas entre 500-420 $\mathrm{cm}^{-1}$ que corresponden a la vibración de los enlaces Si-O y Al-O del tetraedro interno (Van Oers et al., 2014). Por lo anterior, se puede deducir que los picos agudos centrados entre 555 y $463 \mathrm{~cm}^{-1}$ revelan un producto de síntesis de elevada cristalinidad (Gross et al., 2014). Del ensayo de calorimetría de Barrido Diferencial (CBD), se estableció que la temperatura de descomposición del material es aproximadamente $300^{\circ} \mathrm{C}$.

Síntesis de la zeolita tipo Faujasita NaX empleando el silicato de sodio extraído de la diatomita.

\section{Estudio del tiempo de maduración en la relación 1,2.}

Los estudios de DRXP para las muestras obtenidas cuando la relación $\mathrm{Si} / \mathrm{Al}$ fue de 1,2 y el crudo de reacción se sometió a $24 \mathrm{~h}$ de maduración y tiempos de envejecimiento de 0,8 , 16 y $24 \mathrm{~h}$ (DE1, DE2, DE3 y DE4), mostraron que la fase cristalina que se forma en mayor proporción es la zeolita LTA con pequeñas trazas de la zeolita Faujasita $\mathrm{NaX}$, lo cual demuestra que bajo este grado de saturación y con la

Tabla 4. Análisis elemental por fluorescencia de rayos X (FRX) de las anillas.

\begin{tabular}{|c|c|c|c|c|}
\hline Elemento & $\begin{array}{l}\text { Anillas de Aluminio } \\
\text { (AAI) }\end{array}$ & $\begin{array}{l}\text { Anillas calcinadas } \\
\text { (AC) }\end{array}$ & $\begin{array}{c}\text { Residuo extracción } \\
\text { (RE) }\end{array}$ & $\begin{array}{l}\text { Porcentaje de Solubilización } \\
\% S=\left(1-\frac{12,73 g * \% R E}{20,00 g * \% A C}\right) * 100 \%\end{array}$ \\
\hline & Porcentaje (p/p) & Porcentaje (p/p) & Porcentaje (p/p) & Porcentaje (p/p) \\
\hline $\mathrm{Al}$ & $92,65 \%$ & $64,97 \%$ & $9,03 \%$ & $91,15 \%$ \\
\hline $\mathrm{Mg}$ & $4,42 \%$ & $28,48 \%$ & $4,58 \%$ & $89,76 \%$ \\
\hline $\mathrm{Si}$ & $1,39 \%$ & $3,10 \%$ & $0,12 \%$ & $97,54 \%$ \\
\hline $\mathrm{Fe}$ & $0,29 \%$ & $1,03 \%$ & $1,28 \%$ & $20,90 \%$ \\
\hline $\mathrm{Mn}$ & $0,36 \%$ & $0,65 \%$ & $1,69 \%$ & $1,10 \%$ \\
\hline $\mathrm{Ca}$ & $0,16 \%$ & $0,50 \%$ & $0,07 \%$ & $91,09 \%$ \\
\hline $\mathrm{Na}$ & $0,14 \%$ & $0,43 \%$ & $4,29 \%$ & $-535,020 \%$, Se fijaron $0,46 \mathrm{~g}$ \\
\hline K & $0,10 \%$ & $0,24 \%$ & $44 \mathrm{ppm}$ & $88,33 \%$ \\
\hline $\mathrm{Cl}$ & $0,13 \%$ & $0,16 \%$ & $0,00 \%$ & $100,00 \%$ \\
\hline $\mathrm{S}$ & $0,09 \%$ & $0,12 \%$ & $50 \mathrm{ppm}$ & $73,48 \%$ \\
\hline $\mathrm{C}$ & $0,00 \%$ & $0,11 \%$ & $0,00 \%$ & $100,00 \%$ \\
\hline $\mathrm{P}$ & $0,15 \%$ & $0,07 \%$ & $28 \mathrm{ppm}$ & $74,54 \%$ \\
\hline $\mathrm{Zn}$ & $0,02 \%$ & $0,07 \%$ & $0,06 \%$ & $45,44 \%$ \\
\hline $\mathrm{Ga}$ & $0,01 \%$ & $0,02 \%$ & $0,02 \%$ & $36,35 \%$ \\
\hline $\mathrm{Ni}$ & 70ppm & $0,02 \%$ & $0,04 \%$ & $4,53 \%$ \\
\hline V & $0,01 \%$ & $0,01 \%$ & $0,02 \%$ & $4,53 \%$ \\
\hline $\mathrm{Ti}$ & $0,01 \%$ & $0,01 \%$ & $0,03 \%$ & $4,53 \%$ \\
\hline $\mathrm{Cr}$ & 76ppm & 75 ppm & $0,00 \%$ & $100,00 \%$. \\
\hline $\mathrm{Zr}$ & $11 \mathrm{ppm}$ & $27 \mathrm{ppm}$ & $0,00 \%$ & $100,00 \% .$. \\
\hline
\end{tabular}




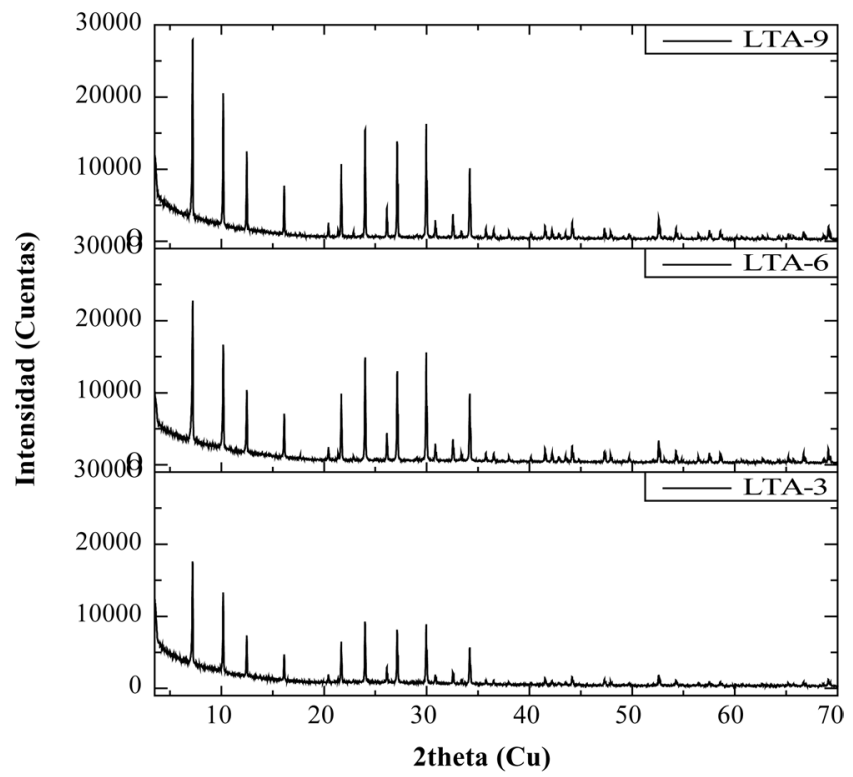

Figura 4. Difractogramas de la zeolita LTA-Na sintetizada, (LTA-3, 3h de envejecimiento; LTA-6 3h de Envejecimiento y LTA-9, 9h de Envejecimiento).

variación del tiempo de envejecimiento no se logra obtener la fase de la $\mathrm{NaX}$ como fase mayoritaria. Con el fin de establecer la influencia de la maduración, se estudiaron los perfiles de las muestras sometidas a $120 \mathrm{~h}$ de maduración y envejecimientos de $0,8,16$ y 24h (DE5, DE6, DE7 y DE8), los cuales, mostraron la presencia de fases zeolitas de la LTA sin la presencia de ninguna traza de Faujasita $\mathrm{NaX}$.

Por lo anterior, se concluye que no es posible obtener la fase de Faujasita $\mathrm{NaX}$ en alta proporción cuando la relación en el crudo de reacción es de 1,2, empleando una solución de $24017,60 \mathrm{ppm}$ de silicato de sodio derivada de diatomita y una solución de $58103,75 \mathrm{ppm}$ de aluminio solubilizado. Por lo cual, se planteó aumentar la relación Si/Al con el fin de saturar el sistema con silicio y así favorecer la formación de cajas más abiertas, facilitando la formación de fases FAUJASITA NaX, lo anterior se fundamenta en que al haber más silicio en el crudo mayor es la capacidad de direccionamiento de los tetraedros de aluminio (Patrikeev et al, 2007).

\section{Relación Si/Al 2,4 en el crudo de reacción y 24 horas de maduración.}

Los perfiles obtenidos cuando el crudo de reacción de la síntesis se mantuvo en una relación $\mathrm{Si} / \mathrm{Al}$ de 2,4 y el proceso de maduración se realizó en $24 \mathrm{~h}$ utilizando tiempos de envejecimiento de $0,8,16$ y 24h (ADE1, ADE2, ADE3, ADE4), muestran que la fase cristalina mayoritaria es la Faujasita NaX con la zeolita LTA-Na en menor proporción. $\mathrm{Al}$ aumentar el tiempo de maduración a $48 \mathrm{~h}$ en la síntesis de estos materiales y aplicar los envejecimientos de $0,8,16$ y 24h (ADE5, ADE6, ADE7 y ADE8), se evidenció que la fase del tipo Faujasita $\mathrm{NaX}$ se forma con menos contaminantes y la fase LTA-Na se forma en muy baja proporción, (Ver Figura 5).

La Figura 6. de la imagen correspondiente a ADE6 muestra la aglomeración de cristales de diferente tamaño alrededor de un cristal de un tamaño superior, de lo que se podría suponer que la fase correspondiente a Faujasita $\mathrm{NaX}$ se encuentra en mayor proporción rodeada por pequeños cristales de LTA. En el sólido ADE8 los cristales observados son de menor tamaño que los observados en el sólido ADE6, notándose que la mayoría de cristales presentes son de diferentes tamaños sustentando los resultados obtenidos por DRXP en donde se evidencia 4 fases para esta muestra.

\subsection{Pruebas de intercambio iónico}

\section{Pruebas de intercambio en la diatomita natural $y$ modificada.}

Al someter muestras de $0,100 \mathrm{~g}$ de diatomita natural y modificada a $40,00 \mathrm{~mL}$ de los nitratos de níquel, cobre y cadmio disueltos a $1000 \mathrm{mg} / \mathrm{L}$, no se presentaron cambios significativos en las concentraciones de las disoluciones mezcladas con los materiales diatomáceos. Por lo que, se puede afirmar que la diatomita natural y modificada bajo la secuencia tres, no presenta fijación o intercambio de los cationes $\mathrm{Ni}^{2+}, \mathrm{Cu}^{2+} \mathrm{y} \mathrm{Cd}^{2+}$ con nitrato como contra anión.

\section{Pruebas de intercambio con las zeolitas LTA-Na y FAUJASITA NaX.}

Al someter cada una de las zeolitas sintetizadas a sus respectivas soluciones de nitrato de níquel, cobre y cadmio, y analizar las alícuotas tomadas cada medio hora por AA se obtuvieron las graficas mostradas en la Figura 7.

De la Figura 7. se puede afirmar que todos los materiales presentan su máxima capacidad de intercambio en los primeros 30 minutos para la mayoría de las zeolitas, lo cual, representa una ventaja en posibles procesos de purificación de aguas contaminadas con cationes como cobre y cadmio, al requerir tiempos bajos de exposición de las soluciones con el aluminosilicato. Por otro lado, se puede establecer que las zeolitas sintetizadas presentan mayor capacidad de intercambio para los cationes cobre (II) y cadmio (II), y baja capacidad de intercambio para el catión níquel (II), tal como se muestra en la Tabla 5.

A partir de los datos mostrados en la Tabla 5 se puede afirmar que las zeolitas con mayor capacidad de fijación de cationes cadmio son la fase FAUJASITA NaX con trazas de LTA-NaX, la cual logra fijar $3,4 \times 10^{-3} \mathrm{~mol} \mathrm{de} \mathrm{Cd}^{2+} / \mathrm{g}$ de material zeolítico, para el catión níquel la mezcla de la muestra ADE6 fija 2,3×10

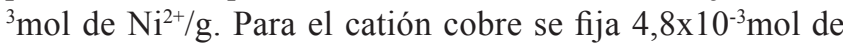




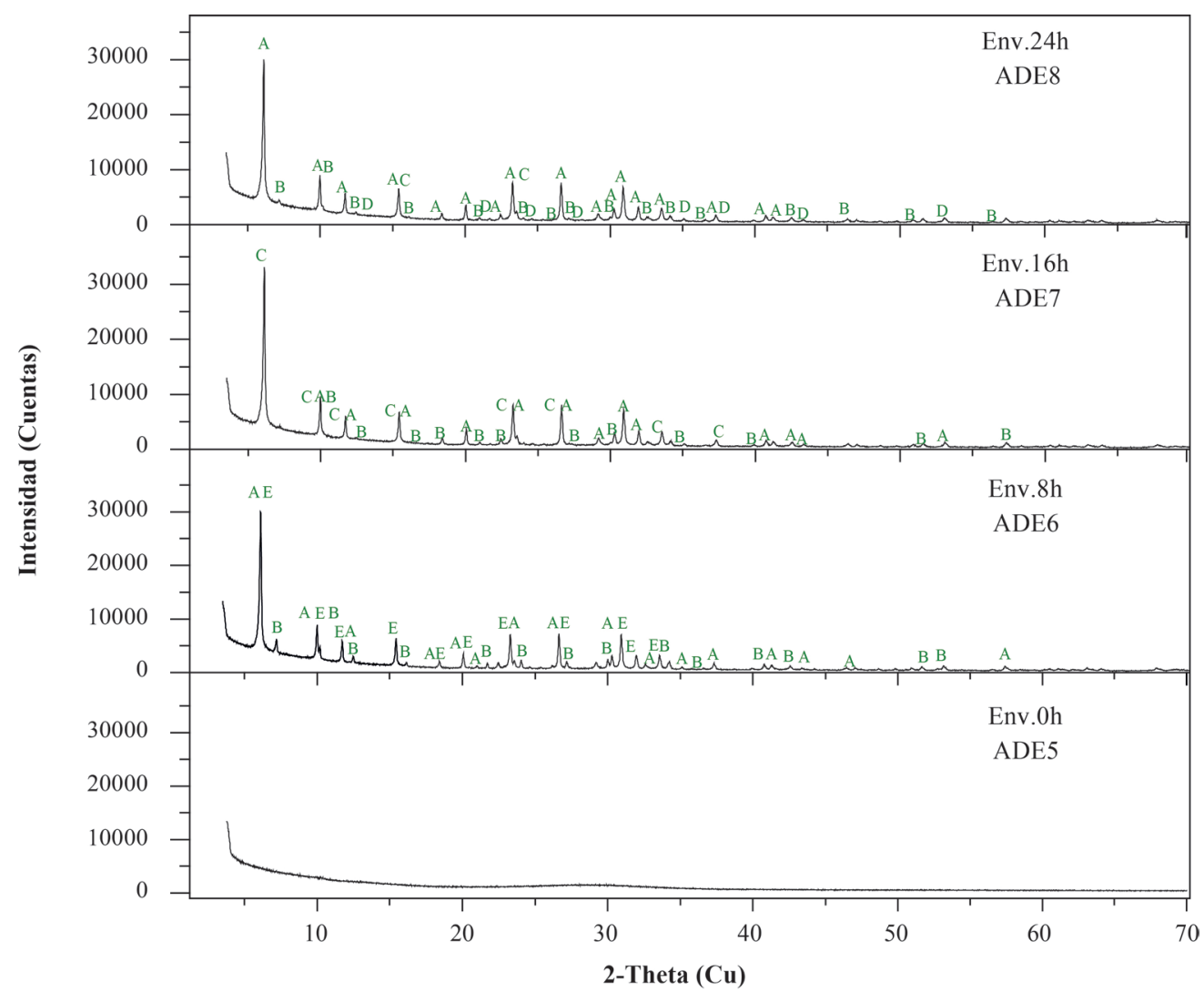

Figura 5. Comparación de los perfiles de DRX con relación Si/Al 2,4, 48h de maduración y variación en los tiempos de envejecimiento, A) $\mathrm{Na}_{2} \mathrm{Al}_{2} \mathrm{Si}_{2.5} \mathrm{O}_{9} * 6_{.2} \mathrm{H}_{2} \mathrm{O}$ (FAU NaX, PDF 38-0237), B) $\mathrm{Na}_{96} \mathrm{Al}_{96} \mathrm{Si}_{96} \mathrm{O}_{384}$ (LTA, PDF 39-0223), C) $\mathrm{Na}_{56.6}\left(\mathrm{Na}_{42.2}\right) \mathrm{Al}_{92} \mathrm{Si}_{100} \mathrm{O}_{384}(\mathrm{FAU}$ NaX, PDF 88-0190), D) $\mathrm{Na}_{96} \mathrm{Al}_{96} \mathrm{Si}_{96} \mathrm{O}_{384} * 2{ }_{16} \mathrm{H}_{2} \mathrm{O}$ (LTA, 39-0222) y E) $\mathrm{Na}_{88} \mathrm{Al}_{88} \mathrm{Si}_{104} \mathrm{O}_{384}{ }^{*} \mathrm{H}_{2} \mathrm{O}$ (FAU NaX, PDF 70-2168).
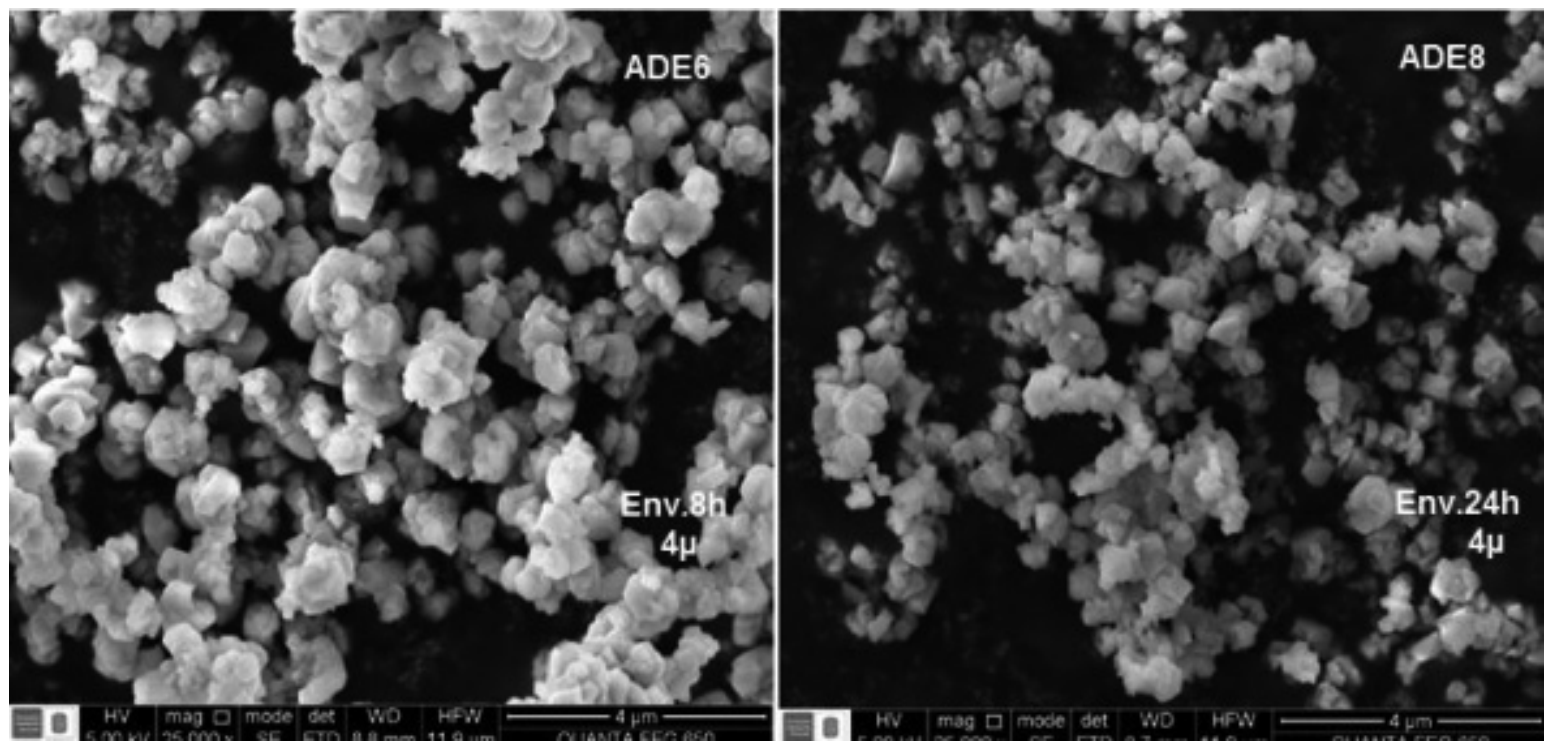

Figura 6. Microscopía electrónica de barrido de la serie con relación Si/Al 2,4 y 48 horas de maduración a partir de la diatomita a 8 (ADE6) y 24 horas de envejecimiento (ADE8). 


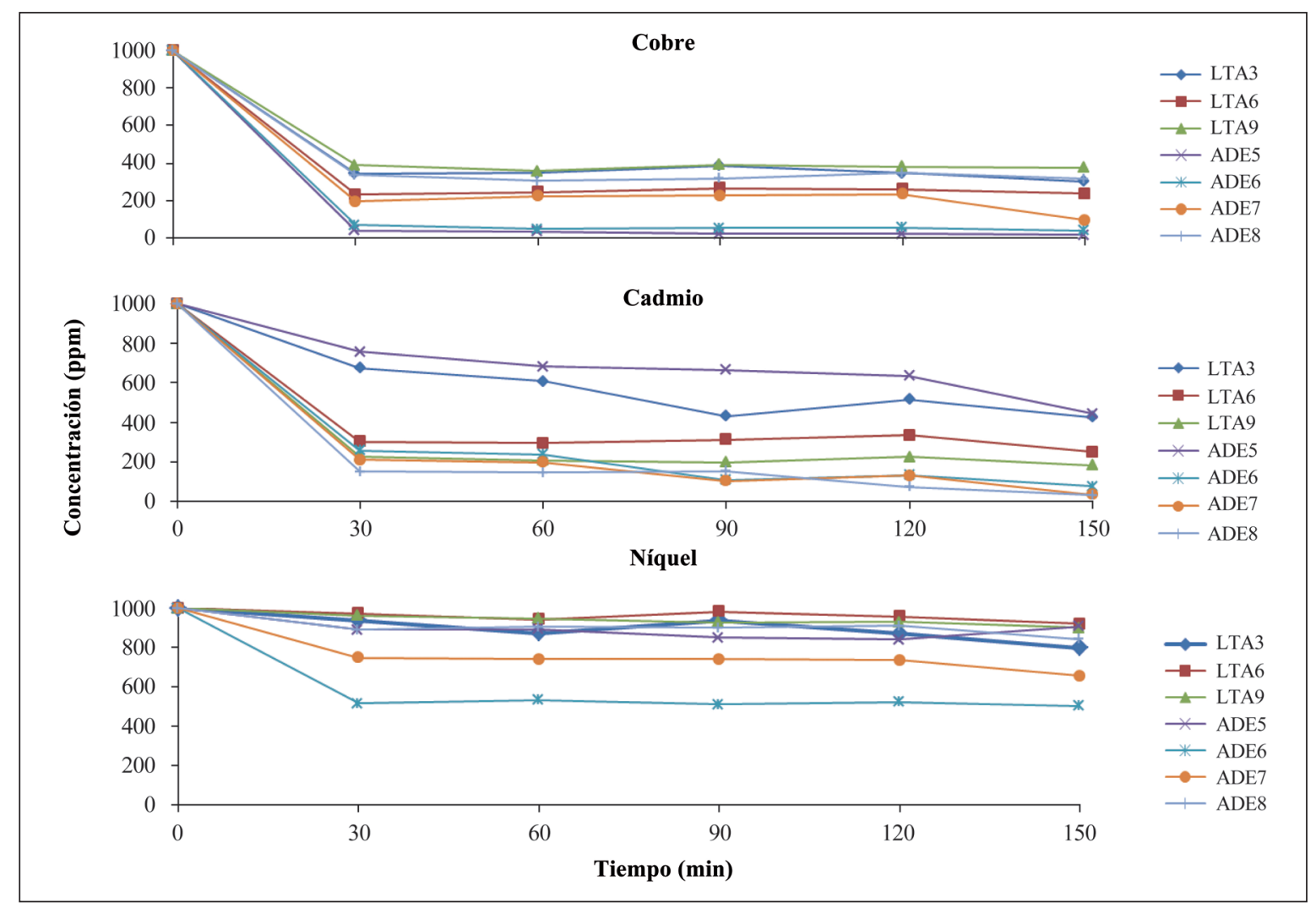

Figura 7. Curvas intercambio iónico para las zeolitas LTA-Na y FAUJASITA NaX.

Tabla 5. Porcentaje de cationes retirados de la soluciones problema y moles fijadas en la zeolitas de $\mathrm{Ni}^{2+}, \mathrm{Cu}^{2+} \mathrm{y} \mathrm{Cd}^{2+}$.

\begin{tabular}{|c|c|c|c|c|c|c|c|}
\hline \multirow{2}{*}{$\begin{array}{c}\text { Tiempo de } \\
\text { Envejecimiento } \\
\text { (horas) }\end{array}$} & \multirow{2}{*}{ Muestra } & \multicolumn{2}{|c|}{ Cadmio (II) } & \multicolumn{2}{|c|}{ Níquel (II) } & \multicolumn{2}{|c|}{ Cobre (II) } \\
\hline & & $\begin{array}{c}\% \text { de } \\
\text { Intercambio }\end{array}$ & $\begin{array}{l}\text { Moles fijadas por } \\
\text { cada 0,1g de zeolita }\end{array}$ & $\begin{array}{c}\% \text { de } \\
\text { Intercambio }\end{array}$ & $\begin{array}{l}\text { Moles fijadas por } \\
\text { cada } 0,1 \mathrm{~g} \text { de zeolita }\end{array}$ & $\begin{array}{c}\% \text { de } \\
\text { Intercambio }\end{array}$ & $\begin{array}{l}\text { Moles fijadas por } \\
\text { cada } 0,1 \mathrm{~g} \text { de zeolita }\end{array}$ \\
\hline 0 & ADE5 & $55,44 \%$ & $2,0 \times 10^{-4}$ & $9,25 \%$ & $4,5 \times 10^{-5}$ & $98,22 \%$ & $4,2 \times 10^{-4}$ \\
\hline 8 & ADE6 & $92,23 \%$ & $3,3 \times 10^{-4}$ & $49,62 \%$ & $2,3 \times 10^{-4}$ & $95,96 \%$ & $4,1 \times 10^{-4}$ \\
\hline 16 & ADE7 & $96,39 \%$ & $3,4 \times 10^{-4}$ & $34,52 \%$ & $1,6 \times 10^{-4}$ & $90,37 \%$ & $3,8 \times 10^{-4}$ \\
\hline 24 & ADE8 & $96,77 \%$ & $3,4 \times 10^{-4}$ & $15,75 \%$ & $7,2 \times 10^{-5}$ & $68,46 \%$ & $2,9 \times 10^{-4}$ \\
\hline 3 & LTA3 & $57,26 \%$ & $2,0 \times 10^{-4}$ & $20,15 \%$ & $1,4 \times 10^{-4}$ & $69,58 \%$ & $4,3 \times 10^{-4}$ \\
\hline 6 & LTA6 & $74,79 \%$ & $2,6 \times 10^{-4}$ & $7,90 \%$ & $5,4 \times 10^{-5}$ & $76,77 \%$ & $4,8 \times 10^{-4}$ \\
\hline 9 & LTA9 & $81,44 \%$ & $2,9 \times 10^{-4}$ & $9,85 \%$ & $6,7 \times 10^{-5}$ & $62,50 \%$ & $3,9 \times 10^{-4}$ \\
\hline
\end{tabular}

$\mathrm{Cu}^{2+} / \mathrm{g}$ de zeolita LTA-Na. Los anteriores resultados son mejores a los publicados por Pehlivan (Pehlivan \& Altun, 2004), donde los autores reportan que por cada $2,4 \times 10^{-3} \mathrm{~mol}$ de $\mathrm{Cd}^{2+} / \mathrm{g}$ de resina DOWEX 50W $(4,8$ mequivalente por $1 \mathrm{~g}$ de resina), lo cual representa el $29,41 \%$ menor al resultado reportado en este trabajo, la misma tendencia se observa para el catión cobre, en el que los autores reportan una fijación de 2,3×10-3 $\mathrm{mol} \mathrm{de} \mathrm{Cu}^{2+} / \mathrm{g}$ de resina DOWEX 50W (4,6mequi/g de resina), dato que representa una fijación menor en un
$52,08 \%$ a la fijación reportada para la muestra LTA-6 en la Tabla 5. Para el catión níquel los resultados reportados en este trabajo son iguales a los de Pehlivan con la resina DOWEX 50W. Otro estudio referencia para el níquel es el reportado por Karthika \& Sekeur en el 2013, en el que los autores estudian dos tipos de resinas modificadas para extraer $\mathrm{Ni}^{2+}$ de soluciones acuosas, reportando que la capacidad de intercambio es de $6,0 \times 10^{-4} \mathrm{~mol} \mathrm{de} \mathrm{Ni}^{2+} / \mathrm{g}$ y $6,80 \times 10^{-4} \mathrm{~mol}$ de $\mathrm{Ni}^{2+} / \mathrm{g}$ para las resinas Amberlite IRC86 y Amberlite 200C 
respectivamente, al comparar estos datos con la capacidad de intercambio de la muestra ADE6 son inferiores en más de un $60 \%$. Lo anterior evidencia la buena capacidad de intercambio de las materiales sintetizados con respecto a las resinas.

Al comparar el comportamiento de las zeolitas LTA con respecto a las FAUJASITAS NaX se puede establecer que la mezcla zeolita FAUJASITA NaX y trazas de LTA presenta mayor capacidad de absorción catiónicas de cadmio y níquel, en el caso del catión cobre, el efecto es contrario, la LTA-Na presenta mayor capacidad de intercambio.

Al medir los perfiles de Difracción de Rayos X de Muestras Policristalinas de cada uno de los materiales zeolíticos después de exponerlos a los intercambios catiónicos, mostraron que las fase zeolíticas se conservan después del intercambio, pero pierden cristalinidad lo que se debe a la reorganización catiónica, la cual afecta los enlaces de condensación en la red cristalina (Salam K., Al-Nasri \& SM Holmes, 2013) para formar fases características a la inclusión de los metales, por ejemplo, para la ADE7 se registraron las siguientes fases $\mathrm{Cd}_{48.96} \mathrm{Si}_{104} \mathrm{Al}_{88} \mathrm{O}_{384 .}\left(\mathrm{H}_{2} \mathrm{O}\right)_{232.64}, \mathrm{Cd}_{57.3}(\mathrm{OH})_{22.6}\left(\mathrm{Al}_{92} \mathrm{Si}_{100} \mathrm{O}_{384}\right)$. $\left(\mathrm{H}_{2} \mathrm{O}\right)_{3.4}, \quad \mathrm{Cd}_{6} \mathrm{Al}_{12} \mathrm{Si}_{12} \mathrm{O}_{48}\left(\mathrm{H}_{2} \mathrm{O}\right)_{6}, \quad \mathrm{Cd}_{55.1}\left(\mathrm{Al}_{92} \mathrm{Si}_{100} \mathrm{O}_{384}\right)(\mathrm{OH})_{18.2 .}$. $\left(\mathrm{H}_{2} \mathrm{O}\right)_{113.9}, \mathrm{Na}_{8} \mathrm{Al}_{9.7} \mathrm{Si}_{26.3} \mathrm{O}_{72},\left(\mathrm{Cd}_{46}\left(\mathrm{Al}_{92} \mathrm{Si}_{100} \mathrm{O}_{384}\right)\right)\left(\mathrm{C}_{2} \mathrm{H}_{4}\right)_{29.5}$, $\mathrm{Cd}_{5.8} \mathrm{Si}_{12} \mathrm{Al}_{12} \mathrm{O}_{48}\left(\mathrm{H}_{2} \mathrm{O}\right)_{2.3}, \mathrm{Na}_{87.30}\left(\mathrm{Al}_{88} \mathrm{Si}_{104} \mathrm{O}_{384}\right) \cdot\left(\mathrm{H}_{2} \mathrm{O}\right)_{32.64}, \mathrm{Cd}_{6}$ $\left(\mathrm{Si}_{12} \mathrm{Al}_{12} \mathrm{O}_{48}\right)$,

\section{Conclusiones}

A partir de la diatomita con especie diatomácea Aulacoseira granulata en mayor proporción y con $\mathrm{SiO}_{2}, \mathrm{Al}_{2} \mathrm{O}_{3}$ y $\mathrm{Fe}_{2} \mathrm{O}_{3}$ en un $71,853 \%, 21,264 \%$ y $3,191 \%$ respectivamente, se logra establecer un proceso de lixiviación en secuencia empleando las soluciones 1. $\mathrm{HCl} 4 \% \mathrm{p} / \mathrm{v}-\mathrm{H}_{2} \mathrm{O}_{4} 2 \% \mathrm{p} / \mathrm{v}, 2 . \mathrm{HNO}_{3}$ $2 \%, \mathrm{p} / \mathrm{v}$ y $3 . \mathrm{H}_{2} \mathrm{SO}_{4} 2 \% \mathrm{p} / \mathrm{v}$ a $90^{\circ} \mathrm{C}, 750 \mathrm{rpm}$, durante $6 \mathrm{~h}$, permite concentrar el $\mathrm{SiO}_{2}$ en la muestra sólida (Diatomita modificada) en un 11,959\%, extrayendo el 57,937\% de $\mathrm{Al}_{2} \mathrm{O}_{3}$ y el $70,844 \%$ de $\mathrm{Fe}_{2} \mathrm{O}_{3}$ presentes en la diatomita natural.

A partir de $4 \mathrm{~g}$ de diatomita modificada sometidas a un tratamiento hidrotérmico con $\mathrm{NaOH} 3 \mathrm{M}$ a $90^{\circ} \mathrm{C}$ y $750 \mathrm{rpm}$, se logra obtener una solución con 24017,60ppm de Silicio (solubilización con un rendimiento del 90,004\%), la cual, mezclada con la solución a 58103,75ppm de aluminio preparada a partir de las uñas de aluminio post-consumo bajo un tratamiento alcalino con $\mathrm{NaOH} 3 \mathrm{M}$ (rendimiento de la reacción del 91,15\%), en una relación $\mathrm{Si} / \mathrm{Al}$ de 1,0 (envejecimiento de 3, 6 y $9 \mathrm{~h}$ a $90^{\circ} \mathrm{C}$ ) y 2,4 (maduración $48 \mathrm{~h}$ y $16 \mathrm{~h}$ de envejecimiento a $90^{\circ} \mathrm{C}$ ), permitió obtener las zeolitas LTA-Na y FAUJASITA NaX con pequeñas trazas de LTA-Na respectivamente. Las zeolitas sintetizadas mostraron buena capacidad de fijación de los cationes $\mathrm{Cu}^{2+}, \mathrm{Ni}^{2+}$ y $\mathrm{Cd}^{2+}$, evidenciando que la mezcla de zeolitas FAUJASITA NaX con trazas de LTA-Na tiene una capacidad de fijación de $2,3 \times 10^{-3} \mathrm{~mol} / \mathrm{g}$ de $\mathrm{Ni}^{2+}$ y $3,4 \times 10^{-}$
${ }^{3} \mathrm{~mol} / \mathrm{g}$ para el $\mathrm{Cd}^{2+}$, mientras que la zeolita LTA-Na

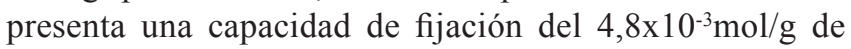
$\mathrm{Cu}^{2+}$, mostrando el potencial de estos materiales para la extracción de metales pesados.

\section{Agradecimientos}

Los autores agradecen a la Vicerectoría de Investigación y Extensión (VIE), a los laboratorios de SEM, RMN y RX del Parque Tecnológico Guatiguará, a Colciencias y la Universidad Industrial de Santander por la beca Jóvenes Investigadores 2013 convocatoria 617.

\section{Bibliografía}

Aleksandrov Y., Tsyganova E. \& Pisarev A. 2003, Reaction of Aluminum with Dilute Aqueous $\mathrm{NaOH}$ Solutions, RUSS. J. GEN. CHEM, 73 (689-694).

Alonso-Zarza A.M. 2010. Petrología sedimentaria. Notas de Teoría. Rocas Silícicas. REDUCA Serie de Petrología Sedimentaria, p. (113-116).

Bahramian B., Ardejani F., Mirkhani V. \& Badii K., 2008. Diatomite-supported manganese Schiff base: An efficient catalyst for oxidation of hydrocarbons, APPL. CATAL. A-GEN. 345 (97-103).

Bernal J.P. \& Railsback L., 2008, Introducción a la tabla periódica de los elementos y sus iones para ciencias de la tierra. REVISTA MEXICANA DE CIENCIAS GEOLÓGICAS. 25 (2) (236-246).

Borai E., Harjula R., Malinen L. \& Paajanen A., 2009, Efficient removal of cesium from low-level radioactive liquid waste using natural and impregnated zeolite minerals, $\mathbf{J}$. HAZARD MATER., 172 (416-422).

Chaisena A., \& Rangsriwatananon K., 2004. Synthesis of sodium zeolites from natural and modified diatomite. MATER LETT 59 (12) (1474-1479).

Danil A., El Gamouz A., Frangie S., Martínez V., Valiente L. \& Webb O., 2012, Turning the volume down on heavy metals using tuned diatomite. A review of diatomite and modified diatomite for the extraction of heavy metals from water, $\mathbf{J}$. HAZARD MATER., 241-242 (14-31).

Davenport W.G., King M.J., Rogers B., Weissenberger A. 2006, Sulphuric Acid Manufacture, SOUTHERN AFRICAN PYROMETALLURGY, 1 (1-16).

Gerengi H., Kocak Y., Jazdzewska A., Kurtay M. \& Durgun H., 2013, Electrochemical investigations on the corrosion behaviour of reinforcing steel in diatomite- and zeolitecontaining concrete exposed to sulphuric acid, CONSTR. BUILD. MATER. 49 (471-477).

Ghosh B., Agrawal D.C., \& Bhatia S., 1994. Synthesis of zeolite A from calcined diatomaceous clay: Optimization studies. IND. ENG. CHEM RES, 33 (9) (2107-2110). 
Gómez, J., Ovejero G. \& Romero M. (2001). Síntesis, caracterización y aplicaciones de las zeolitas básicas. TRABAJO DOCTORAL. Universidad Complutense de Madrid, Madrid, España.

Gross E., Shu X., Alayoglu S., Bechtel H., Martin M., Toste F. \& Somorjai G., 2014. In Situ IR and X-ray High SpatialResolution Microspectroscopy Measurements of Multistep Organic Transformation in Flow Microreactor Catalyzed by Au Nanoclusters, J. AM. CHEM. SOC. 136 (9) (36243629).

Hadjar H., Hamdi B., Jader M., Brendle J., Kessaïssia Z., Balard H. \& Donnet J.B., 2008, Elaboration and characterisation of new mesoporous materials from diatomite and charcoal, MICROPOR. MESOPOR. MAT., 107 (219-226).

Herance, J., Marquet J. \& Bourdelande J., 2005, Reactivos ocluidos en aluminosilicatos: Reactividad y comportamiento en óptica no lineal. Universidad Autónoma de Barcelona, Barcelona, España. TESIS DOCTORAL.

Hollman G.G., Steenbruggen G. \& Janssen-Jurkovičová M., 1999. A two-step process for the synthesis of zeolites from coal fly ash. FUEL 78 (10), 1225-1230.

Insung O., Ter Meulen U. \& Langholz H., 1999. Use of diatomite filter aid residue in cattle feed. Georg-August-University Göttingen, Göttingen, Germany. Ph.D THESIS

Karthika C. \& Sekar M., 2013. Comparison studies of Adsorption Properties on Ni(II) Removal by Strong and Weak acid Cation-exchange Resins, RES. J. CHEM. SCI. 3 (3) (65-69).

Kumar V., Matsuda M. \& Miyake M., 2008, Sorption properties of the activated carbon-zeolite composite prepared from coal fly ash for $\mathrm{Ni}^{2+}, \mathrm{Cu}^{2+} \mathrm{Cd}^{2+}$ and $\mathrm{Pb}^{2+}$, J. HAZARD. MATER., 160 (148-153).

Liu D., Yuan W., Deng L., Yu W., Sun. H. \& Yuan P., 2014, Preparation of porous diatomite-templated carbons with large adsorption capacity and mesoporous zeolite K-H as a byproduct, COLLOID INTERFACE SCIENCE, Accepted Manuscript.

Liu H., Lu G., Guo Y., Guo Yun \& Wang J., 2004. Effect of pretreatment on properties of TS-1/diatomite catalyst for hydroxylation of phenol by $\mathrm{H}_{2} \mathrm{O}_{2}$ in fixed-bed reactor. CATAL. TODAY. 93-95 (353-357).

Lopez P.J., Desclés J., Allen A.E. \& Bowler Ch. 2005. Prospects in diatoms research. CURR OPIN BIOTECH, 16 (2) (180-186).

Mohamedbakr H., \& Burkitbaev, M. 2009. Elaboration and Characterization of Natural Diatomite in Aktyubinsk/ Kazakhstan. THE OPEN MINERALOGY JOURNAL 3 (1) (12-16)

Mohamedbark, (2010). Diatomite: Its Characterization, Modifications and Applications. ASIAN J MAT. SCI. 2 (3) (121-136).

Naranjo W., Gaviria S., \& Manosalva S. 2007. Mineralógica y Geoquímica de Diatomitas (Boyacá, Colombia). GEOLOGÍA COLOMBIANA 32 (77-88).
Oliva M., Ramírez J., Garduño G., Cañétas J., \& Ortega M. 2005. Caracterización diatomológica de tres cuerpos de agua de los humedales de Jilotepec-Xitlahuaca, Estado de México. HIDROBIOLÓGICA 15 (1) (1-26).

Patrikeev V.A., Pavlov M.L., Kutepov B.I., Makhamatkhanov R.A., Travkina., Sthestopal A.L. \& Dzhemilev U.M., 2007. Crystallization of X-Type Zeolite from Concentrated Sodium Silicate and Aluminate Solutions. BRIEF COMMUNICATIONS 80 (3) (498-500).

Pehlivan E. \& Altun T., 2006, The study of various parameters affecting the ion exchange of $\mathrm{Cu}^{2+}, \mathrm{Zn}^{2+}, \mathrm{Ni}^{2+}, \mathrm{Cd}^{2+}$, and $\mathrm{Pb}^{2+}$ from aqueous solution on Dowex 50W synthetic resin, $\mathbf{J}$. HAZARD. MATER., B134 (149-156).

Pozo M. \& López M.J., 2004. Facies biosilíceas en el neógeno de la cuenca de madrid: origen e implicaciones sedimentológicas. REVISTA DE LA SOCIEDAD GEOLÓGICA DE ESPAÑA 17 (3-4) (229-248).

Qiu W. \& Zheng Y., 2009, Removal of lead, copper, nickel, cobalt, and zinc from water by a cancrinite-type zeolite synthesized from fly ash, CHEM. ENG. J., 145 (483-488).

Rangsriwatananon, K., Chaisena, A., \& Thongkasam, Ch. 2008. Thermal and acid treatment on raw natural diatomite influencing on synthesis of sodium zeolites. JOURNAL OF POROUS MATERIALS 15 (5) (499-5059.

Rodríguez A., Romero J. \& Castro E. 2005. Comportamiento refractario de probetas de morteros conformadas con diatomitas como agregado mineral. REVISTA COLOMBIANA DE QUÍMICA 34 (2) (139-146).

Salam K., Al-Nasri \& SM Holmes, 2013. Effect of Different Conditions on the Sorption Behaavior of $\mathrm{Co}^{2+}$ Using Celatom-Zeolite Y Compisite, MATER SCI ENG, Vol. 7 No $9(1-7)$.

Shan W., Zhang Y., Wang Y., Xia J. \& Tang Y., 2004. Synthesis of Meso-/Macroporous Zeolite (Fe,Al)-ZSM-5 Microspheres from Diatomite, CHEM LETT, 33 (3) (270-271).

Van Oers C.J., Góra-Marek K., Sadowska K., Mertens M., Meynen V., Datka J. \& Cool P., 2014. In situ IR spectroscopic study to reveal the impact of the synthesis conditions of zeolite $\boldsymbol{\beta}$ nanoparticles on the acidic properties of the resulting zeolite, CHEM. ENG. J. 237 (372-379).

Villavicencio C., Molina A., \& Fernández L. (2009). Estudio de la adsorción sobre zeolitas sintéticas modificadas con surfactantes. REVISTA DE LA FACULTAD DE INGENIERÍA U.C.V. 24 (3) (95-1079.

Wajima T. \& Munakata K., 2012, Synthesis of Zeolitic Material from Paper Sludge Ash Using Diatomite, MATERIALS TRANSACTIONS, 53 (4) (592-596).

Wang S. \& Peng Y., 2010, Natural zeolites as effective adsorbents in water and wastewater treatment, CHEM. ENG. J., 156 (11-24). 
Widiastuti. N., Wu H., Ang H. M., Dongke Z, 2011, Removal of ammonium from greywater using, DESALINATION, 277 (15-23).

Yang X., Yang S., Yang S., Hu J., Tan X. \& Wang X., 2011. Effect of $\mathrm{pH}$, ionic strength and temperature on sorption of $\mathrm{Pb}$ (II) on NKF-6 zeolite studied by batch technique, CHEM. ENG. J. 168 (86-93).

Yilmaz B., \& Ediz N., 2008. The use of raw and calcined diatomite in cement production. CEMENT AND CONCRETE COMPOSITES 30 (3), 202-211.
Yi-qui., Lei Z. \& Xin-you Z., 2012. Investigation of low-temperature properties of diatomite-modified asphalt mixtures, CONSTR. BUILD. MATER., 36 (787-795).

Zhang K., Liu Y., Tian S., Zhao E., Enhong Z., Zhang J \& Liu C., 2013. Preparation of bifunctional $\mathrm{NiPb} / \mathrm{ZnO}$-diatomiteZSM-5 catalyst and its reactive adsorption desulfurization coupling aromatization performance in FCC gasoline upgrading process, FUEL, 104 (201-207). 